\title{
Psicologia e interfaces pedagógicas \\ Análise da aprendizagem mediada por uma interface educativa voltada para a resolução de situações aditivas com suporte diagramático
}

\author{
Ana Emilia de Melo Queiroz \\ Alex Sandro Gomes \\ Cláudia Roberta Araújo Gomes \\ Soraia Oliveira Mota Barreto
}

\section{SciELO Books / SciELO Livros / SciELO Libros}

QUEIROZ, A.E.M., GOMES, A.S., GOMES, C.R.A., and BARRETO, S.O.M. Análise da aprendizagem mediada por uma interface educativa voltada para a resolução de situações aditivas com suporte diagramático. In: SANTIAGO, A.M.S., and FONSÊCA, A.L.B., comp. Psicologia e suas interfaces: estudos interdisciplinares [online]. Salvador: EDUFBA, 2016, pp. 185-218. ISBN 978-85-232-2007-5. https://doi.org/10.7476/9788523220075.0008.

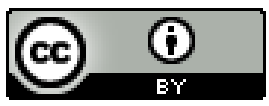

All the contents of this work, except where otherwise noted, is licensed under a Creative Commons Attribution 4.0 International license.

Todo o conteúdo deste trabalho, exceto quando houver ressalva, é publicado sob a licença Creative Commons Atribição 4.0. 


\section{Análise da aprendizagem mediada por uma interface educativa voltada para a resolução de situações aditivas com suporte diagramático}

Ana Emilia de Melo Queiroz, Alex Sandro Gomes, Cláudia Roberta Araújo Gomes, Soraia Oliveira Mota Barreto

Introdução

A representação e suas diferentes expressões têm um papel fundamental na resolução de problemas matemáticos. (GOLDIN, 1998a, 1998b, 2003; GOLDIN; KAPUT, 1996; KAPUT, 1993)

Neste estudo, estamos interessados nas diferentes expressões da representação utilizada no ensino das Estruturas Aditivas. (ELIA; GAGATSIS; DEMETRIOU, 2007; GAGATSIS, 2004; GAGATSIS; ILIADA, 2005; HARRIES; BARMBY, 2007; HARRIES; SUGGATE, 2006; MICHAELIDOU; GAGATSIS, 2004; SELVA; ROCHA; NUNES, 2005) Para tanto, encontramos na literatura a forma decorativa, a pictorial, a figura informativa, a reta numérica, a descrição textual, os vetores, 
a superfície retangular, os gráficos cartesianos, os blocos de tijolos, o gráfico de barras, os contadores, os blocos estruturados como unidades e como dezenas, as contas, o dinheiro, a seta, os cartões e os quadrados numerados. Ou seja, são várias as expressões da representação para os conceitos presentes nas Estruturas Aditivas. Entretanto, Gagatsis e Iliada (2007, p. 45, tradução nossa ) observam em Elia, Gagatsis e Demetriou (2007) que "cada sistema representacional apresenta suas próprias regularidades" e, por esse motivo, elas apresentam aspectos diferentes do mesmo conceito.

As representações acima citadas foram utilizadas com crianças em idades variadas para efetuar tarefas de reconhecimento do conceito, representação do conceito, translação de conceitos entre formas (ELIA; GAGATSIS; DEMETRIOU, 2007), comparação de medidas, composição de medida (SELVA; ROCHA; NUNES, 2005), transformação aplicada a uma medida (ELIA; GAGATSIS; DEMETRIOU, 2007) e para representar números. (HARRIES; SUGGATE, 2006)

Assim como nas iniciativas acima citadas, temos particular interesse em observar a tarefa de resolução de situações aditivas no uso de uma forma de representação. Entretanto, posicionamos esse trabalho nas iniciativas de educação à distancia voltadas para a formação continuada de professores. Ou seja, um cenário no qual existe a figura do professor aprendiz, aquele que deseja aprender para refletir e, em função dessa reflexão, melhor transpor os conceitos matemáticos e compreender as necessidades dos seus alunos.

Para um cenário como esse, a representação escolhida foi a legenda de Vergnaud (1986). Ela foi especificamente desenvolvida para cenários de ensino da Matemática (VERGNAUD, 1998) que envolvem formação continuada de professores. O meio material é uma interface educativa interativa e os usuários finais são os professores polivalentes que lecionam nas séries iniciais do ensino fundamental I. O motivo para a escolha da legenda se deveu ao fato de que, numa situação-problema, um conceito nunca aparece isolado, e, no uso dos diagramas, é possível vivenciar uma experiência na qual não apenas 
um conceito é representado mas as relações que por ventura existam, entre eles, também serão representadas e passíveis de serem percebidas no curso da ação.

O objetivo deste trabalho é, portanto, observar o impacto que a interação dos usuários-professores, com a forma de representação diagramática, teve sobre o processo de resolução de problemas. Para alcançar tal objetivo, a tarefa de resolução dos problemas foi analisada segundo um modelo teórico que serve para realizar testes de usabilidade de interfaces educativas voltado para avaliar qualitativamente o impacto de uma interface à aprendizagem de conceitos específicos ainda durante sua criação. (GOMES, 2008) A escolha dessa metodologia foi motivada pela necessidade de se entender o processo por meio do qual a tarefa era executada, ou seja: subtarefas, ações, operações, artefatos utilizados e estilo de interação. Ou seja, identificar os elementos que estiveram presentes nas ações dos usuários - professores em situações nas quais houve verbalização de proposições verdadeiras sobre os conceitos veiculados.

\section{Diagramas no ensino de estruturas aditivas}

Quando se considera que diferentes tipos de problemas podem ser oferecidos visando a compreensão por parte dos sujeitos, o campo conceitual das Estruturas Aditivas pode ser categorizado da seguinte forma: composição de medidas, comparação de medidas e transformação aplicada a uma medida (como exemplificadas no Quadro 2, abaixo). Entretanto, apenas a representação “ $4+7$ = 11” não reflete as relações que estão inseridas no contexo de cada situação-problema. Por esse motivo, Verganud $(1986,1998)$ propõe em seus estudos, acerca dos campos conceituais, uma tríade que representaria o significado de um conceito, são essas:

1) a situação;

2) a representação; e

3) os invariantes operatórios. 
Nesse contexto, as situações-problema (1) são narrativas como exemplificadas no Quadro 2. A representação (2), por sua vez, é composta pelos elementos presentes no Quadro 1 e constitui-se em uma ferramenta que favorece a percepção das relações existentes entre os significados da situação-problema. Assim sendo, espera-se que tal percepção se dê por meio da construção de um diagrama - cujo esquema também foi proposto por Vergnaud (1986) e que possibilita a execução tanto do cálculo numérico quanto do cálculo relacional, favorecendo e evidenciando as diferenças entre as situações. Os inviariantes operatórios (3) são proposições sobre os números presentes nas situações-problema. À medida que o usuário-professor constrói os diagramas, ele é exposto a situações que podem favorecer a percepção de invariantes operatórios sobre os conceitos estudados, uma vez que identificar os invariantes operatórios e relacioná-los ao uso da interface possibilita acompanhar a aprendizagem de conceitos que ocorre como consequencia desse uso. (GOMES, 2008)

\section{Quadro 1 - Forma de representação dos conceitos}

\begin{tabular}{|c|c|}
\hline LEGENDA & FUNÇÃO OU DESCRIÇÃO \\
\hline\{ & $\begin{array}{l}\text { Representar a categoria de composição } \\
\text { demedidas ou grandezas. }\end{array}$ \\
\hline & Representar uma medida ou um número natural. \\
\hline & Representar o número relativo. \\
\hline$\Leftrightarrow$ & Representar a categoria de transformação. \\
\hline$\uparrow$ & Representa a categoria de comparação. \\
\hline
\end{tabular}

Fonte: Elaborados pelos autores. 
Quadro 2 - Categoria da situação-problema e sua representação diagramática correspondente

\begin{tabular}{|c|c|c|c|}
\hline CATEGORIA & $\begin{array}{l}\text { SOLUÇÃO } \\
\text { NUMÉRICA }\end{array}$ & $\begin{array}{l}\text { REPRESENTAÇÃO } \\
\text { DA SITUACCÃO- } \\
\text { PROBLEMA }\end{array}$ & $\begin{array}{l}\text { REPRESENTAÇÃO } \\
\text { DA CATEGORIA }\end{array}$ \\
\hline $\begin{array}{l}\text { I. Composição de medidas } \\
\text { Ao redor da mesa da sala de jantar } \\
\text { de minha casa, estão sentados } \\
\text { apenas } 4 \text { garotos e } 7 \text { garotas. } \\
\text { Quantas pessoas estão sentadas ao } \\
\text { redor da mesa? } \\
\text { São duas quantidades, que } \\
\text { estão expressas, de existência } \\
\text { concomitante, a partir das quais } \\
\text { o indivíduo deve compor uma } \\
\text { terceira quantidade. }\end{array}$ & $4+7=11$ & $\left.\begin{array}{l}4 \\
7\end{array}\right\}^{l}$ & 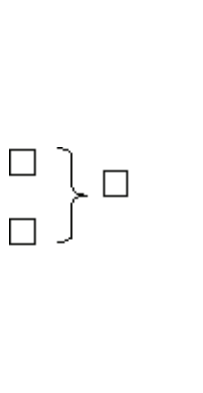 \\
\hline $\begin{array}{l}\text { II. Transformação de medida } \\
\text { Maria comprou uma caixa de } \\
\text { bombons por } \mathrm{R} \$ 4,00 \text { e ainda ficou } \\
\text { com R\$ } 7,00 \text {. Quanto ela possuía } \\
\text { antes de fazer a compra? } \\
\text { As quantidades iniciais e finais } \\
\text { são conhecidas, mas, no caso, o } \\
\text { que se deseja saber é o valor da } \\
\text { transformação que ocorre entre o } \\
\text { primeiro e o segundo momento. }\end{array}$ & $4+7=11$ & $2 \stackrel{-(4)}{\longrightarrow}$ & $\square \stackrel{\bigcirc}{\longrightarrow} \square$ \\
\hline $\begin{array}{l}\text { III. Comparação de medidas } \\
\text { Carlos tem } 4 \text { anos. Maria é } 7 \text { anos } \\
\text { mais velha que Carlos. Quantos } \\
\text { anos tem Maria? } \\
\text { Mais uma vez, as quantidades } \\
\text { são conhecidas e concomitantes, } \\
\text { buscando-se comparar a diferença } \\
\text { existente entre as duas (relação). }\end{array}$ & $4+7=11$ & $\prod_{4}^{?}+7$ & $\uparrow_{\square}^{\square}$ \\
\hline
\end{tabular}

Fonte: Elaborado pelos autores.

Como no Quadro 2, no caso de composição de medidas, os elementos numéricos presentes na situação problema são posicionados dentro dos quadrados pertencentes ao diagrama e, no item desconhecido, pode ser colocada uma interrogação. A partir disso, o diagrama passa a representar a situação-problema com seus aspectos qualitativos e quantitativos. Em situações como essa, variam-se as quantidades e o local valor do item desconhecido, e tais variações 
conduzem a diagramas diferentes ajudando o usuário-professor a perceber que as situações-problema podem variar. O diagrama é, portanto, um tipo de ajuda ou suporte, com caminho predefinido, que possibilita ao usuário-professor refletir sobre suas ideias, conduzindo-o à compreensão da estrutura profunda do problema.

\section{Ações dos usuário e da interface}

Nesse contexto, as ações dos professores aprendizes são executadas por meio de tarefas de mouse, que, no total, somam seis (SHNEIDERMAN, 1998):

1) Selecionar: o usuário escolhe um item a partir de um conjunto de itens;

2) Posicionar: o usuário escolhe um ponto em um espaço de uma ou mais dimensões;

3) Orientar: o usuário escolhe um ponto em um espaço de duas ou mais dimenões;

4) Quantificar: o usuário especifica um valor numérico;

5) Caminho: o usuário executa rapidamente tarefas de posionar e orientar;

6) Texto: o usuário entra, modifica, move, e edita textos em um espaço de duas dimensões.

Enquanto o usuário constrói os diagramas por meio das tarefas de mouse, a interface oferece alguns feedbacks, os quais são denominados Scaffolding. (SEDIG; KLAWE; WESTROM, 2001; SOUZA; SEDIG, 2001; WOOD; BRUNNER; ROSS, 1971) Nesse contexto, a interface pode oferecer os seguintes feedbacks:

1) Feedback instrucional: são mensagens que oferecem informações sobre as discrepâncias entre as ações realizadas sobre o material e aquelas consideradas corretas; 
2) Automatização de passos com tarefa completa: realizada para reduzir o grau de liberdade, o que implica na simplificação da tarefa. Tal simplificação é feita por meio da redução do número de ações realizadas sobre o meio material para obter a solução da situação-problema. Nesse contexto, a interface ainda oferece uma demonstração ou solução para a tarefa;

3) Feedback de questionamento: nesse tipo de Scaffolding, a interface pergunta para o usuários se ele tem certeza de que está seguindo o caminho correto;

4) Metáfora de material concreto: a interface oferece um tipo de Scaffolding que se caracteriza pela execução de uma tarefa similar a que está sendo executada. Entretanto, no uso de metáforas de material concreto.

Nesse contexto, foram utilizados quadradinhos manipuláveis para executar contagem.

Em outras palavras, em cada ação, o usuário-professor pode utilizar as tarefas de mouse para efeutar point and click, drag and drop e preenchimento de formulário. Além disso, ele pode receber o suporte da interface. Esse conjunto de estilos de interação é denominado interface WIMP ${ }^{1}$ com Manipulação Direta Reflexiva de Conceitos (MDRC). Nessa modalidade de interação, além dos elementos presentes na interface WIMP, ocorre o oferecimento de um feedback visual, construído por meio de uma representação metafórica do conceito. Entretanto, esse feedback deve ser gradativamente removido, à medida que se tornar desnecessário para o usuário-professor, ou seja, quando ele não precisar mais daquele tipo de feedback. (SEDIG; KLAWE; WESTROM, 2001; SOUZA; SEDIG, 2001; WOOD; BRUNNER; ROSS, 1971)

1 Padrão para Windows, Icon, Menu, Pointing device, denotando o estilo de interação que oferece ao usuário esses elementos de interação. 
À luz dessas discussões anteriores, elaboramos as seguintes questões:

1) Quais elementos icônicos estiveram presentes?

a) Quais elementos da interface estiveram disponíveis para serem manipulados e aqueles que não estiveram?

i) Quais as tarefas de mouse foram potencialmente utilizados na manipulação dos elementos da forma?

ii) Qual a relação entre as tarefas de mouse utilizados nas ações e os invariantes operatórios?

b) Qual o impacto dessa disponibilidade sobre a organização das ações dos professores?

2) Qual a relação entre as regras de ação e os invariantes operatórios mobilizados pelo usuário durante a resolução da tarefa?

3) Qual a influência do plano de ações do usuário sobre a interface?

\section{Procedimento}

Cinco usuários, descritos no Quadro 4, foram gravados e filmados enquanto falavam sobre suas ações durante a resolução das situações-problemas (Quadro 3) numa interface em papel. (SNYDER, 2003) Dessas, cada usuário-professor resolveu quatro situações- problemas, sendo exploradas as categorias das estruturas aditivas presentes no Quadro 2, quais sejam: composição de medidas, comparação de medidas e transformação aplicada a uma medida. Posteriormente, mais uma foi escolhida de forma aleatória entre as seis. Ao final da resolução de cada situação-problema, os vídeos foram mostrados com o objetivo de ser introduzida uma entrevista semiestruturada focal (FLICK, 2004) visando conhecer as explicações dos usuários sobre os motivos de suas ações. Ao final da resolução de 
quatro situações-problema, realizou-se uma entrevista cujo propósito foi receber informações gerais com as impressões dos usuários na vivência. A duração média das entrevistas foi de 60 a 90 minutos por participante.

Quadro 3 - Situações-problema

\begin{tabular}{|llllll|}
\hline USUÁRIO - PROFESSOR & 01 & 02 & 03 & 04 & 05 \\
\hline Sexo & $\mathrm{M}$ & $\mathrm{F}$ & $\mathrm{M}$ & $\mathrm{F}$ & $\mathrm{F}$ \\
Idade & 45 & 30 & 48 & 30 & 35 \\
Escolaridade & $\mathrm{PG}$ & $2^{\circ}$ & $\mathrm{PG}$ & $\mathrm{PG}$ & $\mathrm{PG}$ \\
Formação & $\mathrm{MA}$ & $\mathrm{MG}$ & $\mathrm{MA}$ & $\mathrm{MA}$ & $\mathrm{MA}$ \\
Séries que leciona & $\mathrm{EF}$ & $\mathrm{EF}$ & $\mathrm{EF}$ & $\mathrm{EF}$ & $\mathrm{EF}$ \\
Tipos de escola & $\mathrm{P}$ & $\mathrm{PU}$ & $\mathrm{P}$ & $\mathrm{P}$ & $\mathrm{P}$ \\
\hline
\end{tabular}

Legenda: CP: Composição de medidas, TM: Transformação de medidas, COP: Comparação de medidas; ID: Identificador.

Fonte: Elaborado pelos autores.

Quadro 4 - Análise da aprendizagem mediada por uma interface educativa voltada para resolução situações aditivas com suporte diagramático

\begin{tabular}{|c|c|c|}
\hline ID & SITUAÇÃO-PROBLEMA & Tipo \\
\hline P1 & $\begin{array}{l}\text { Ao redor da mesa estão sentados } 4 \text { garotos e } 7 \text { garotas. Quantas pessoas estão } \\
\text { sentadas ao redor da mesa? }\end{array}$ & $\mathrm{CP}$ \\
\hline P2 & $\begin{array}{l}\text { Ricardo saiu de casa para jogar com } 6 \text { bolas de gude. Ao voltar ele possuía } 2 \text { bolas. } \\
0 \text { que aconteceu no jogo? }\end{array}$ & TM \\
\hline P3 & $\begin{array}{l}\text { Maria comprou uma caixa de bombons por } 4 \text { reais e ainda ficou com } 4 \text { reais. } \\
\text { Quanto ela possuía antes de fazer a compra? }\end{array}$ & TM \\
\hline P4 & $\begin{array}{l}\text { Ricardo tem } 6 \text { anos, Carlos tem } 4 \text { anos a mais do que ele. Quantos anos tem } \\
\text { Carlos? }\end{array}$ & COP \\
\hline P5 & Carlos tem 7 reais e Luiz tem 6 reais a menos do que ele. Quantos reais tem Luiz? & TM \\
\hline P6 & $\begin{array}{l}\text { Ricardo tem } 9 \text { brinquedos dos quais uma parte são carrinhos e a outra parte são } \\
\text { bonecos. Sabendo que existem } 4 \text { bonecos, quantos são os carrinhos? }\end{array}$ & $\mathrm{CP}$ \\
\hline
\end{tabular}

Legenda: PG: Pós-Graduação, MA: Matemática, EF: Ensino Fundamental, PU: Pública, P: Particular. Fonte: Elaborado pelos autores. 
Para resolver as situações-problema presentes no Quadro 3, existe uma organização prévia, a qual foi exemplificada na Figura 1. Entretanto, à medida que ele inicia o processo, pode cometer alguns erros e, para cada ação, especificamente, o usuário-professor pode apresentar uma organização própria, construída com base em algum conhecimento prévio ou por tentativa e erro. Inicialmente, focamos nosso olhar apenas nos erros relativos à construção do diagrama. Contudo, existem outros tipos de erro, que podem estar associados à inabilidade com o uso da interface - por exemplo, existem usuários que não sabem usar o mouse. Nesse estudo, os erros dessa natureza não foram considerados.

Figura 1 - Análise da tarefa

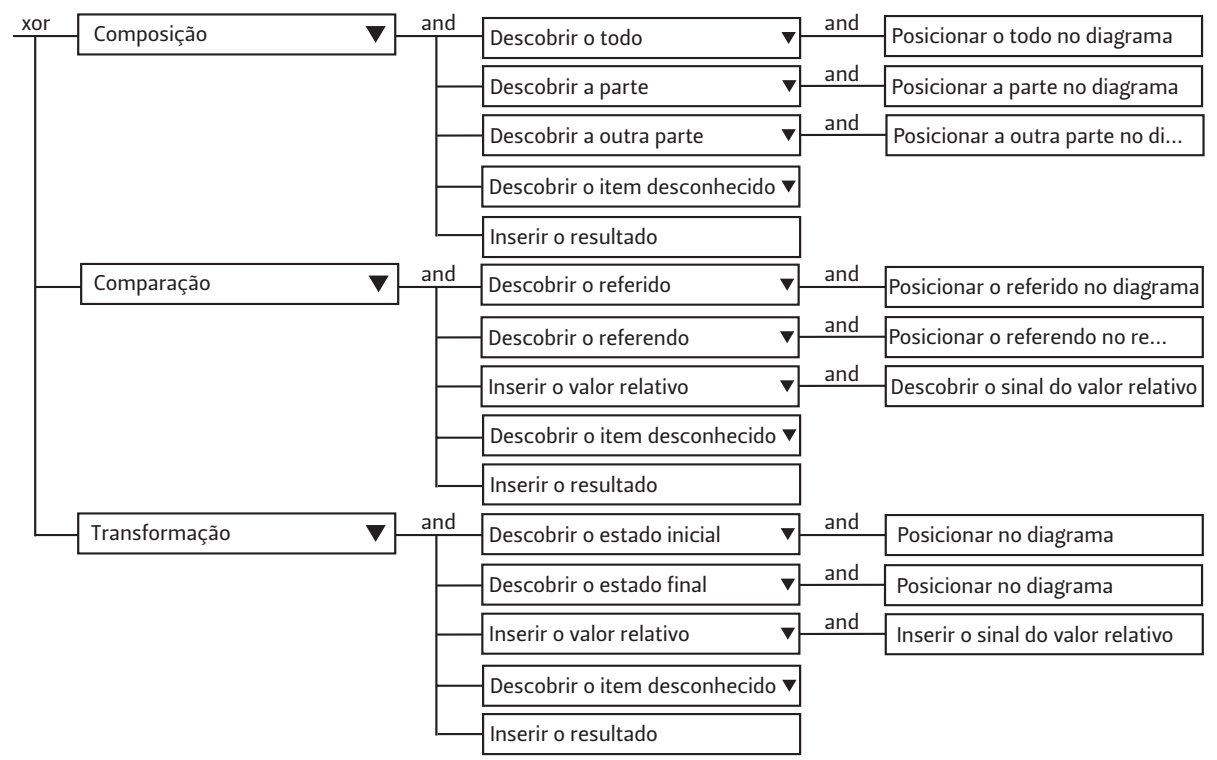

Fonte: Elaborada pelos autores.

A tarefa de construção dos diagramas é composta por um conjunto de ações como dispostas na Figura 1. Exemplificando com um problema de transformação de medidas, o usuário-professor precisa identificar corretamente a categoria, em seguida, os elementos do 
texto implicados no problema e encontrar sua adequada posição no diagrama. Como visto na Figura 1, existe uma organização que pode ser seguida para construção do diagrama. A primeira ação a ser realizada é escolher no menu a adequada categoria a qual pertence o problema. Para cada problema só pode haver uma categoria, ou ele é de comparação, ou de composição, ou de transformação, não havendo intersecção entre essas categorias. Essa ausência de intersecção é denotada pelo anotação "xor" no canto esquerdo da Figura 1, que significa uma relação na qual apenas um dos três itens é verdadeiro. Em seguida, ele inicia o processo de identificação de elementos e posicionamento no diagrama. Tal processo é apoiado pelo estilo de interação oferecido na interface. A partir desses elementos iniciais, foi contruído um protótipo em linguagem de programação JAVA, como apresentado na Figura 2.

Figura 2 - Gerard implementado em linguagem JAVA

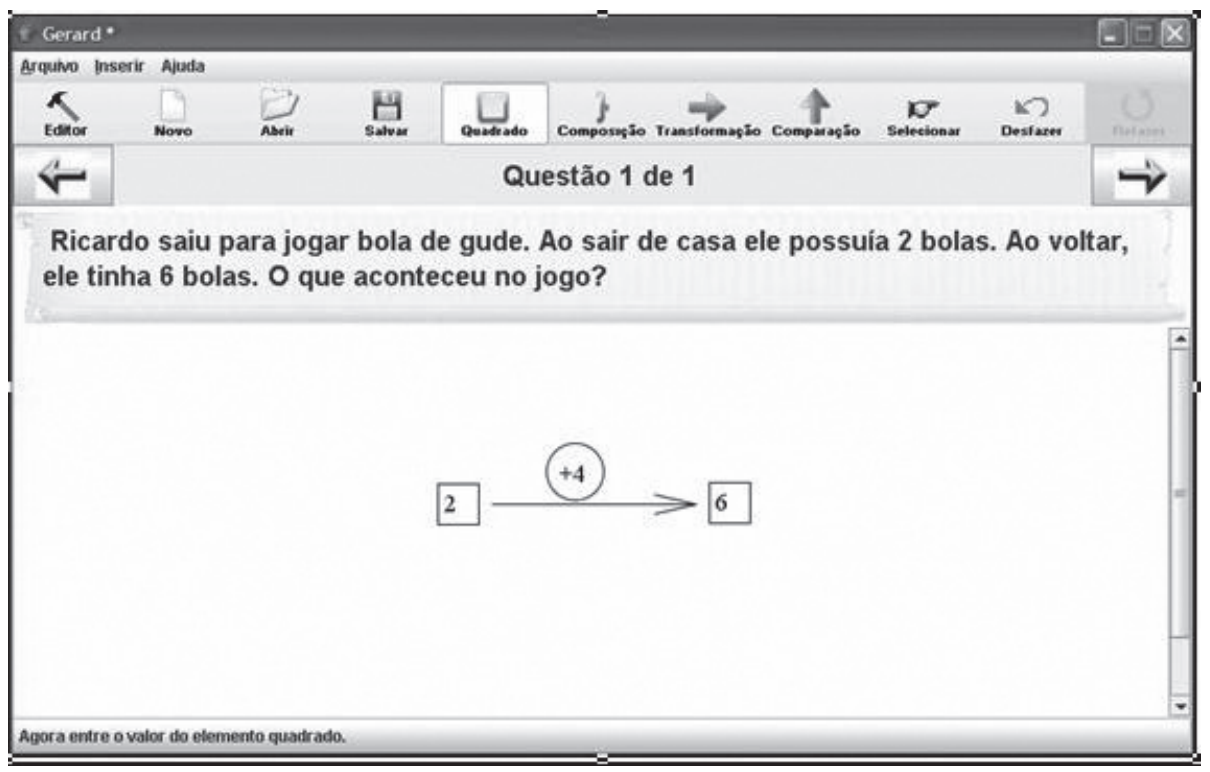

Fonte: Braga, Queiroz e Gomes (2008, p. 9). 
Figura 3 - Protótipo da interface em papel, construído a partir da Figura 2
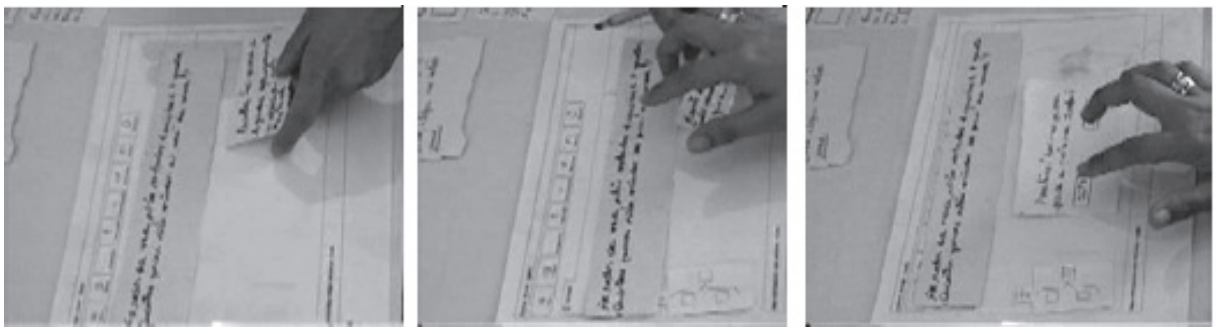

Fonte: Elaborada pelos autores.

A partir da interface presente na Figura 2, foi construído o protótipo em papel da Figura 3. Segundo a literatura, a prototipação em papel é uma variação do teste de usabilidade, no qual usuários representativos interagem com uma versão da interface em papel para realizar tarefas reais. Tal interface é manipulada por uma pessoa, que simula o computador, e reage a todas as ações do usuário na interface, sem contudo conduzi-las. Sendo assim, esse método não usa bancos de dados, redes ou servidores, eliminando, por esse motivo, muitas das situações que podem causar problema. (SNYDER, 2003) Além disso, com baixo investimento, a prototipação em papel possibilita uma rápida externalização das ideias de design. (BAILEY et al., 2007)

O protótipo em papel apresenta-se, ainda, como um elemento facilitador da interação e seu uso visa minimizar os erros relativos à inabilidade com o uso de interfaces computacionais, favorecendo que os usuários sintam-se mais à vontade para expressar suas opiniões. A partir desse cenário, aqui descrito e apresentado na Figura 3, restanos, agora, confrontar a prévia análise da tarefa presente na Figura 1 com a que foi observada durante o experimento.

Sempre que um usuário-professor executava uma tarefa de mouse (SHNEIDERMAN, 1998) - selecionar, posicionar, orientar, quantificar, caminho e texto - ou quando a interface oferecia algum feedback (SEDIG; KLAWE; WESTROM, 2001; SOUZA; SEDIG, 2001; WOOD; BRUNNER; ROSS, 1971), uma ação era contabilizada. A fim de analisar cada ação, notadamente, no que diz respeito à emergência de inva- 
riantes operatórios, utilizamos um modelo teórico de base construtivista (GOMES, 2008) para oferecer resultados que relacionam o uso da interface educativa com a emergência de tais invariantes. Ao todo foram contabilizadas 584 ações instrumentais para os usuários do Quadro 4, resolvendo os problemas do Quadro 3. Segue um extrato dos resultados do usuário-professor S1, resolvendo o problema P2.

Figura 4 - Plano de ações do usuário-professor S1 no problema P2

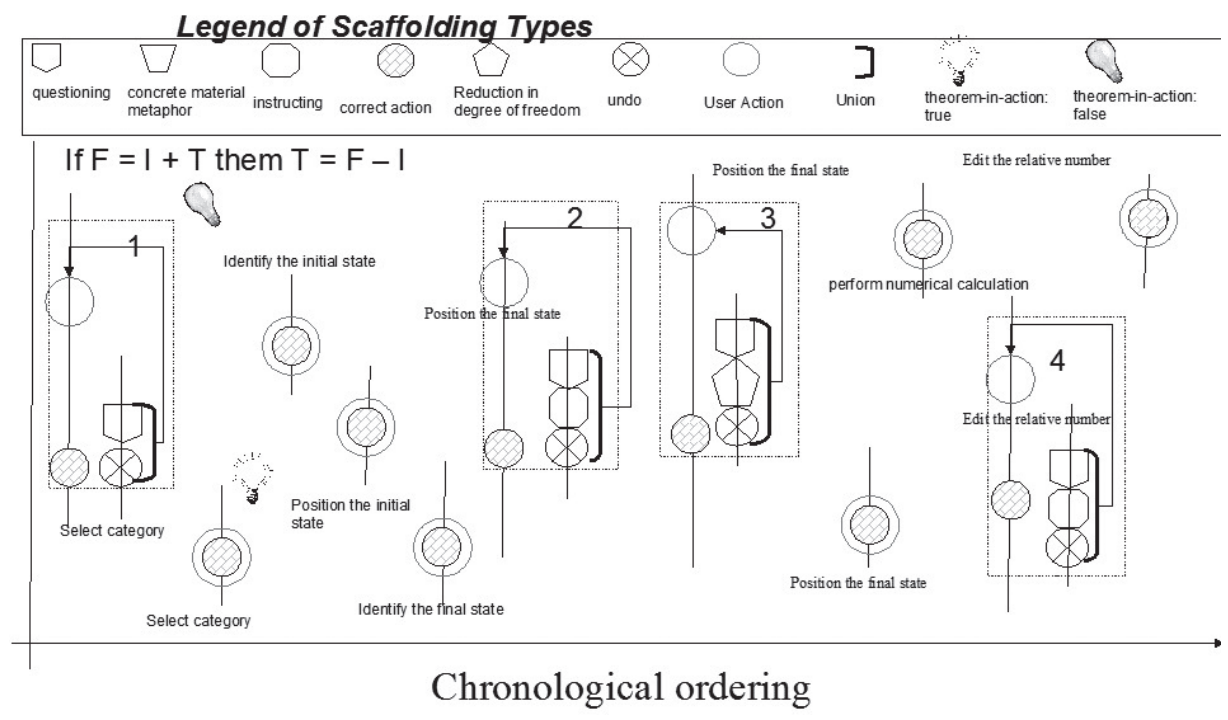

Fonte: Elaborada pelos autores.

Na Figura 4 é mostrado um plano de ações executado pelo usuário-professor S1 para resolver o problema P2. Para esse problema, o invariante operatório é: "Se F = I + T então T = F - I". Ou seja, se o estado final é igual ao estado inicial somado ao valor correspondente à transformação (número relativo), então a transformação (ou número relativo) é igual ao estado final menos o estado inicial.

As áreas hachuradas 1, 2, 3 e 4 exemplificam o uso inadequado do diagrama, ou seja, momentos nos quais os usuários cometeram erro de posicionamento. Mais especificamente, na área hachurada 1, o usuário-professor inicia a construção do diagrama errando a primeira ação (escolha da categoria à qual pertence a situação-problema). Em se- 
guida, ele recebe uma ajuda do tipo questionamento e executa quatro ações corretas. Na área hachurada 2, o usuário identifica e seleciona corretamente o elemento no texto, mas o posiciona de maneira incorreta no diagrama. Percebido o erro, a interface ofereceu um feedback em formato texto, que, além de não ter sido suficiente para esclarecer o problema, ainda causou uma má interpretação, fazendo-o persistir no erro de posicionamento. Na área hachurada 3 , contudo, o usuário modifica o sinal do número, pois, após receber um feedback inadequado na área hachurada 2, passou a acreditar que o erro de sinal era o único erro cometido. Ainda na área hachurada 3, ele recebe duas ajudas: a primeira reduz o grau de liberdade da ação e a segunda faz um questionamento. Após isso, o usuário executa duas ações corretas. Concluindo, na área hachurada 4, ele, ainda, comete mais um erro de omissão de sinal do número relativo.

A cada ação, do usuário-professor ou do computador, a interface assume um novo estado. Os estados gerados e registrados na execução das ações, como mostradas na Figura 4, são insumos a partir dos quais foi possível identificar os invariantes operatórios do Quadro 5. Dessa forma, foram identificados 16 invariantes operatórios.

O Quadro 5 apresenta um conjunto de 16 invariantes operatórios que foram identificados durante o uso da interface. Esses invariantes são proposições sobre o domínio e podem ser verdadeiros ou falsos. A partir deles é possível observar se ocorre predominância desses associados a determinados conceitos. Ou seja, tendo o conjunto de invariantes mobilizados na ação, pode-se oferecer resultados qualitativos tanto sobre a aprendizagem de conceitos quanto como relacioná-los ao uso da interface computacional (o que é apresentado no Quadro 6). 


\begin{tabular}{|c|c|c|c|}
\hline VALOR & IDENTIFICAÇÃO & INVARIANTE OPERATÓRIO & CONCEITO \\
\hline v & Inv_1 & As partes formam o todo. & \\
\hline V & Inv_2 & $\begin{array}{l}\text { A soma das quantidades gera um valor que } \\
\text { corresponde à cardinalidade do todo. }\end{array}$ & Composição \\
\hline v & Inv_3 & & \multirow{6}{*}{$\begin{array}{l}\text { Transformação } \\
\text { e conceito de } \\
\text { número }\end{array}$} \\
\hline v & Inv_4 & $\begin{array}{l}\text { Uma medida inicial se transforma, dando } \\
\text { origem a uma nova medida. }\end{array}$ & \\
\hline v & Inv_5 & $\begin{array}{l}\text { Um número pode representar o estado inicial } \\
\text { da medida. }\end{array}$ & \\
\hline v & Inv_6 & $\begin{array}{l}\text { Um número pode representar o estado final da } \\
\text { medida. }\end{array}$ & \\
\hline v & Inv_7 & $\begin{array}{l}\text { A transformação aplicada a uma medida pode } \\
\text { ser expressa por um número. }\end{array}$ & \\
\hline v & Inv_8 & $\begin{array}{l}\text { A transformação aplicada a uma medida é } \\
\text { obtida pela diferença entre o estado final e o } \\
\text { estado inicial da mesma. }\end{array}$ & \\
\hline v & Inv_9 & $\begin{array}{l}\text { O estado inicial em um problema de } \\
\text { transformação pode ser obtido a partir da } \\
\text { aplicação da transformação inversa ao estado } \\
\text { final da medida. }\end{array}$ & \multirow[t]{2}{*}{$\begin{array}{l}\text { Transformação } \\
\text { e comparação }\end{array}$} \\
\hline v & Inv_10 & $\begin{array}{l}\text { O valor de uma medida é especificado em relação } \\
\text { ao valor de uma outra medida, em termos de } \\
\text { acréscimos ou decréscimos. }\end{array}$ & \\
\hline v & Inv_11 & $\begin{array}{l}\text { Um número pode representar o referente de uma } \\
\text { medida, servindo de base para a obtenção da } \\
\text { outra medida. }\end{array}$ & \multirow{4}{*}{ Comparação } \\
\hline v & Inv_12 & $\begin{array}{l}\text { A relação existente entre as duas medidas pode } \\
\text { ser expressa por um número. }\end{array}$ & \\
\hline $\mathrm{F}$ & Inv_13 & $\begin{array}{l}0 \text { cardinal do referendo é obtido pela composição } \\
\text { entre o número relativo e o cardinal do referido. } \\
\text { (Falso) }\end{array}$ & \\
\hline v & Inv_14 & $\begin{array}{l}0 \text { referido em um problema de comparação } \\
\text { é obtido a partir da soma do referente com o } \\
\text { número que expressa a relação existente entre o } \\
\text { referente e o referido. }\end{array}$ & \\
\hline v & Inv_15 & $\begin{array}{l}\text { O número representa a relação existente entre as } \\
\text { duas medidas. }\end{array}$ & \multirow{2}{*}{$\begin{array}{l}\text { Conceito de } \\
\text { número }\end{array}$} \\
\hline & Inv_16 & $\begin{array}{l}\text { Um número pode representar o cardinal de um } \\
\text { conjunto. }\end{array}$ & \\
\hline
\end{tabular}

Fonte: Braga, Queiroz e Gomes (2008). 
Quadro 6 - Análise das quatro situações para o usuário-professor S1

\begin{tabular}{|c|c|c|c|c|}
\hline \multirow{2}{*}{ TE } & \multicolumn{2}{|l|}{ ARTEFATO } & \multirow{2}{*}{ REGRA DE AÇÃO } & \multirow{2}{*}{ T.e.A. } \\
\hline & PROPRIEDADE & COMPOSIÇÃO & & \\
\hline 01 & $\begin{array}{l}\text { Números manipuláveis que representam } \\
\text { os cardinais dos conjuntos. } \\
\text { Uma chave, dois quadrados, um do } \\
\text { lado direito da chave e outro do lado } \\
\text { esquerdo, denotando a existência } \\
\text { concomitante de dois conjuntos que se } \\
\text { unem e formam um único conjunto. }\end{array}$ & $\begin{array}{l}\text { Três quadrados, } \\
\text { uma chave. }\end{array}$ & Posicionamento & Inv_16 \\
\hline 02 & $\begin{array}{l}\text { Números manipuláveis que representam } \\
\text { os cardinais dos conjuntos. } \\
\text { Uma seta para direita. Um quadrado no } \\
\text { lado esquerdo da seta, e outro no lado } \\
\text { direito, dentando uma relação entre } \\
\text { a quantidade inicial e a quantidade } \\
\text { final. Um círculo no meio da seta } \\
\text { representando essa relação. }\end{array}$ & $\begin{array}{l}\text { Dois quadrados, } \\
\text { um círculo, uma } \\
\text { seta horizontal. }\end{array}$ & $\begin{array}{l}\text { Posicionamento } \\
\text { Inserção de sinal }\end{array}$ & $\begin{array}{l}\text { Inv_6 } \\
\text { Inv_5 }\end{array}$ \\
\hline 03 & $\begin{array}{l}\text { Números manipuláveis que representam } \\
\text { os cardinais dos conjuntos. } \\
\text { Uma seta vertical apontando para cima. } \\
\text { Um quadrado na parte inferior e outra } \\
\text { na parte superior, denotando que existe } \\
\text { um ponto de referência, a partir do qual } \\
\text { uma relação é estabelecida. Um círculo } \\
\text { no meio para representar essa relação. }\end{array}$ & $\begin{array}{l}\text { Dois quadrados, } \\
\text { um círculo, uma } \\
\text { seta vertical que } \\
\text { representa. }\end{array}$ & $\begin{array}{l}\text { Posicionamento } \\
\text { Inserção de sinal }\end{array}$ & $\begin{array}{l}\text { Inv_16 } \\
\text { Inv_12 }\end{array}$ \\
\hline 04 & Os mesmos da tentativa 02 & $\begin{array}{l}\text { Os mesmos da } \\
\text { tentativa } 02\end{array}$ & $\begin{array}{l}\text { Posicionamento } \\
\text { Inserção de sinal }\end{array}$ & $\begin{array}{l}\text { Inv_6 } \\
\text { Inv_15 }\end{array}$ \\
\hline
\end{tabular}

Legenda: TE - Tentativa.

Fonte: Elaborado pelos autores.

Como pode ser visto no Quadro 6, os artefatos, nas quatro situações, apresentam, em sua maior parte, componentes que representam cardinais de números e números relativos. Os elementos da forma de maior prevalência representam o conceito de número, e, na manipulação desses, foram utilizadas as tarefas de mouse "Posicionar" e "Texto". Os invariantes operatórios de maior prevalência são justamente aqueles relacionados ao conceito de número. As setas, horizontal e vertical, bem como a chave, não foram manipuladas 
pelo usuário-professor. A inserção desses componentes na interface aconteceu de maneira automática.

Além das regularidades presentes no Quadro 6, foi possível mostrar outra forma de visualização, uma perspectiva quantitativa cujo propósito foi identificar as tarefas mais difíceis para os usuários, e, em cada tarefa, as etapas nas quais houve maior prevalência de erro.

Quadro 7 - Resumo quantitativo dos passos no curso das tarefas para o usuárioprofessor S1

\begin{tabular}{|cclccc|}
\hline TE & SP & ERROS COMETIDOS & NP & NF & NC \\
\hline 1 & P1 & Nenhum & 09 & 0 & 1 \\
2 & P2 & $\begin{array}{l}\text { Erro de categorização; erro de } \\
\text { posicionamento; erro de sinal }\end{array}$ & 22 & 8 & 1 \\
3 & P4 & Erro de posicionamento & 11 & 2 & 1 \\
4 & P3 & Nenhum & 09 & 0 & 1 \\
\hline
\end{tabular}

Legenda: TE - Tentativa, SP - Situação-problema, NP - Número total de passos, NF - Número de Feedbacks, NC - Número de passos realizados pelo computador.

Fonte: Elaborado pelos autores.

A ordem de resolução das tarefas para o usuário-professor S1 foi essa que está presente no Quadro 7. Por exemplo, na tarefa P2, o usuário-professor precisou de 22 passos e recebeu 8 feedbacks da interface. Já na tarefa P3, pertencente à mesma categoria da situação-problema P2, o usuário não cometeu nenhum erro e no seu plano de ações evidenciou-se procedimentos de antecipação (QUEIROZ; GOMES, 2008), mostrando que ele lembrou da ajuda recebida na execução da tarefa P2 bem como de sua finalidade, o que pode ter colaborado na execução da situação-problema P3.

Para todas as situações-problema, sintetizamos os quantitativos de ações, realizadas pelos usuários e pelo computador. 
Quadro 8 - Número total de ações (TA) por situação-problema

\begin{tabular}{|ll|}
\hline SITUAÇÃO-PROBLEMA & $\mathrm{N}^{\circ}$ TA \\
\hline P1 & 69 \\
P2 & 72 \\
P6 & 77 \\
P4 & 98 \\
P5 & 106 \\
P3 & 162 \\
TOTAL & 584 \\
\hline
\end{tabular}

Fonte: Elaborado pelos autores.

No Quadro 8, aparece a situação-problema e, ao seu lado, o número de passos necessários na sua execução. Entretanto essas tarefas são formadas por etapas, e, nesse ponto, vale salientar que é necessário entender melhor quais as etapas de cada tarefa foram mais difíceis para os usuários, bem como os feedbacks mais efetivos na ultrapassagem de tais dificuldades.

Quadro 9 - Etapas na tarefa ordenadas por número de erros

\begin{tabular}{|c|c|c|}
\hline ETAPAS DA TAREFA & PREDOMINÂNCIA & ESTRATÉGIA UTILIZADA \\
\hline Erro de sinal na transformação & $\mathrm{P} 2$ & Feedback instrucional \\
\hline $\begin{array}{l}\text { Erros consecutivos de } \\
\text { posicionamento }\end{array}$ & $\mathrm{P} 2, \mathrm{P} 3, \mathrm{P} 4$ & $\begin{array}{l}\text { Automatização de passos com dica } \\
\text { completa }\end{array}$ \\
\hline Erro de posicionamento & P5, P3, P6 & $\begin{array}{l}\text { Automatização de passos com dica } \\
\text { completa }\end{array}$ \\
\hline Erros de categorização & & Feedback de questionamento \\
\hline Erro de sinal na comparação & & Feedback instrucional \\
\hline Erro de cálculo numérico & & Feedback de questionamento \\
\hline Erro consecutivo de categorizacão & $\mathrm{P} 2, \mathrm{P} 4$ & Feedback de questionamento \\
\hline
\end{tabular}

Fonte: Elaborado pelos autores. 
Na visão do Quadro 10, pretendeu-se identificar quais as etapas em cada tarefa foram mais difíceis para os usuários, bem como os tipos de feedbacks mais apropriados em cada etapa. Nesses momentos, além dos erros, observou-se a ocorrência de algumas necessidades que já haviam sido previstas, além de mais algumas novas, não previstas no planejamento inicial

\section{Quadro 10 - Contingências observadas durante as tarefas}

\begin{tabular}{|ll|}
\hline CONTINGÊNCIAS OBSERVADAS & ESTRATÉGIA UTILIZADA \\
\hline $\begin{array}{l}\text { Necessidade de saber quais elementos } \\
\text { do texto deveriam ser arrastados para o } \\
\text { diagrama. }\end{array}$ & $\begin{array}{l}\text { Apontar os elementos do texto e do diagrama } \\
\text { com uma caneta. }\end{array}$ \\
$\begin{array}{l}\text { Antecipações: tentativa de negligenciar os } \\
\text { passos da tarefa, ou seja, alguns usuários } \\
\text { tentaram colocar o resultado sem construir } \\
\text { o diagrama. }\end{array}$ & $\begin{array}{l}\text { Informar que apenas os elementos presentes } \\
\text { no texto poderiam ser arrastados para o }\end{array}$ \\
$\begin{array}{l}\text { Antecipações: correção do erro cometido } \\
\text { sem receber ajuda. }\end{array}$ & Sem estratégia. \\
$\begin{array}{l}\text { Conclusão com sucesso. } \\
\text { Necessidade de saber qual o próximo passo } \\
\text { da tarefa. }\end{array}$ & $\begin{array}{l}\text { Betão contendo o rótulo "Qual o próximo } \\
\text { passo?". }\end{array}$ \\
Necessidade de pedir dica. & Botão contendo o rótulo "Mais dica". \\
\hline
\end{tabular}

Fonte: Elaborado pelos autores.

O Quadro 10 apresenta algumas modificações incorporadas à interface em consequência das contingências observadas. Para algumas delas, já havia uma previsão advinda dos estudos piloto. Para outras, não havíamos feito nenhuma previsão. Por exemplo, o tipo de antecipação voltada para negligenciar a forma não havia sido prevista.

\section{Discussão}

Inicialmente, Queiroz e Gomes (2008) analisaram a influência que os feedbacks ou Scaffoldings tiveram sobre a resolução das situações- problema, notadamente no que diz respeito a verificação de indícios 
de generalização e desenvolvimento de autonomia no curso da tarefa. Partindo desses resultados, buscamos, neste trabalho, estabelecer relações entre a forma de representação, os modos de manipulação e a emergência de proposições verdadeiras sobre o campo conceitual estudado com os usuários da Quadro 4. Ou seja, buscamos perceber relações qualitavas entre os elementos do Quadro 1, Quadro 2, Quadro 3 e Quadro 5, as quais encontram-se evidenciadas no Quadro 5. Tais resultados são requisitos não funcionais sobre aprendizagem de conceitos específicos e, em razão disso, eles são insumos a partir dos quais serão modeladas situações interativas mais ajustadas às tarefas do Quadro 3 e aos usuários do Quadro 4. Tais situações serão oferecidas aos usuários por meio da interface educativa apresentada na Figura 2 e Figura 3.

\section{Elementos da forma e o impacto de sua disponibilidade na organização das ações dos professores}

A forma de representação adotada neste estudo foi a legenda de Vergnaud (1986), bem como os diagramas construídos no uso de tais elementos. Como mostrado no Quadro 1, cada elemento da forma é utilizado para representar um conceito. Entretanto cabe verificar quão exposto a tais conceitos o usuário-professor esteve à medida que construiu os diagramas. Em outras palavras, quais as ações que puderam ser realizadas pelo usuário-professor com a forma e quais aquelas que não puderam. A fim de tornar mais clara a exposição do usuário a tais elementos, apresentamos no Quadro 11 uma visão matricial, contendo, na linha, os elementos do diagrama e, na coluna, as tarefas de mouse potencialmente utilizadas. 
Quadro 11 - Ui: manipulado diretamente pelo usuário-professor havendo emergência de invariantes operatórios. C: manipulado pelo computador

\begin{tabular}{|c|c|c|c|c|c|c|}
\hline & POSICIONAR & TEXTO & SELECIONAR & ORIENTAÇÃO & CAMINHO & QUANTIFICAR \\
\hline\{ & C & & & & & \\
\hline & $U_{i}$ & $U_{i}$ & & & & \\
\hline & $U_{i}$ & $U_{i}$ & & & & \\
\hline & c & & & & & \\
\hline$\Rightarrow$ & & & & & & \\
\hline & c & & & & & \\
\hline$\uparrow$ & & & & & & \\
\hline
\end{tabular}

Fonte: Elaborado pelos autores.

Nas linhas, está a forma de representação do conceito, nas colunas, estão as tarefas de mouse. As células preenchidas indicam que houve manipulação da forma segundo a tarefa disposta na coluna. As células vazias denotam ausência de manipulação da forma segundo a tarefa de mouse presente na coluna: Posicionar, Texto, Selecionar, Orientação, Caminho, Quantificar, Ui: manipulado diretamente pelo usuário-professor havendo emergência de invariantes operatórios. C: manipulado pelo computador.

O Quadro 11 mostra as formas de representação do conceito juntamente com o tipo de manipulação direta sobre essas. Como pode ser visto, três desses elementos não foram manipulados diretamente pelos usuários. Eles foram inseridos ou posicionados na interface automaticamente, por meio de uma ação advinda do computador. Além disso, naqueles elementos sobre os quais houve manipulação do usuário-professor, essa foi realizada por meio das tarefas de mouse de "Posicionar" e "Texto". Mais especificamente, cabe pontuar que o quadrado e o círculo não foram eles próprios manipulados, mas serviram, no entanto, como delimitadores da ação. Ou seja, os elementos manipulados foram os objetos 
presentes na ação: os números que representam os cardinais dos conjuntos que figuram nas situações-problema. Entretanto, esses números foram posicionados dentro do quadrado e dentro do círculo como pode ser visto no Quadro 2. Nessa associação, do objeto com a forma, deu-se a emergência de invariantes operatórios como descrita em detalhes na coluna cinco do Quadro 6. Essa verificação traz implicações para estudos futuros, tendo em vista que os outros elementos - a seta vertical, horizontal e a chave - não foram manipuladas diretamente pelos usuários.

Além disso, ainda caberia verificar quais os tipos de tarefas de mouse seriam mais adequadas nas situações: Selecionar, Orientação, Caminho e Quantificar. Mais particularmente, caberia identificar quem, dos atores, presentes no contexto - computador ou usuário - professor - seria mais apropriado manipular a forma e, além disso, em quais as situações tal manipulação poderia ocorrer. Ou seja, caberia verificar: (i) quais elementos da forma seriam mais apropriados para serem manipulados (SOUZA; SEDIG, 2001), (ii) através de quais tarefas de mouse (SOUZA; SEDIG, 2001) e (iii) qual seria o ator mais apropriado para manipular a forma - computador ou usuário. Para tanto, é necessário se obter informações como as presentes no Quadro 6 e oferecer resultados qualitativos para todas as combinações de elementos da forma versus tarefas de mouse. Em outras palavras, seria necessário completar integralmente o Quadro 11. Feito isso, seria possível inferir, mais especificamente, dentre os invariantes operatórios presentes no Quadro 5, quais deles tiveram maior prevalência em quais circunstâncias da situação interativa. Sabendo disso, seria possível classificar as situações interativas, para que, juntamente com a classificação dos usuários, segundo sua habilidade no trato com o conteúdo, fossem ofertadas situações-interativas (situações-problema com algum grau de interatividade) mais ajustadas a cada usuário-professor e a cada situação-problema especificamente.

Ainda no que diz respeito às tarefas realizadas com formas, em tarefas de reconhecimento e representação de conceitos sobre número 
decimais, a forma que obteve melhor desempenho foi a superfície retangular. (MICHAELIDOU; GAGATSIS, 2004) Na tarefa de translação, o melhor desempenho foi a partir da reta numérica para o sistema simbólico, comparativamente a translação feita do sistema simbólico para a reta numérica. Entretanto, tais resultados foram conseguidos em contextos específicos, nos quais havia uma intenção comparativa entre formas para determinadas tarefas. Além disso, a habilidade dos alunos em resolver situações-problema é altamente relacionada com a habilidade já desenvolvida para interagir com a forma de representação (ELIA; GAGATSIS; DEMETRIOU, 2007), ou seja, quando eles não souberam usar as formas de representação, caiu o desempenho no trato com as situações-problema. Complementando, os autores apontam forte relacionamento entre o uso de formas de representação combinado com a semântica da situação-problema proposta, ou estrutura do problema.

No sentido de analisar as dificuldades dos alunos na resolução de tarefas com a reta numérica, Harries e Barmby sugeriam em Harries e Suggate (2006) que tais dificuldades repousam no fato de que existem duas operações que devem ser executadas sobre a linha: marcar os pontos e executar os ciclos, e, na direção contrária, para interpretar o ciclo, é preciso ler os pontos marcados. Como as formas representam aspectos diferentes do mesmo conceito, os erros em cada forma também foram analisados. Por exemplo, os mesmos autores analisaram os diferentes tipos de erros cometidos sobre as representações: eles observaram que alguns deles se repetiram em formas de representações diferentes. Além disso, observaram a frequência de determinados erros nos estudantes. Em alguns casos, o erro foi associado a uma ação errada e não necessariamente um erro conceitual. (HARRIES; SUGGATE, 2006) A análise dos erros pode, portanto, indicar os aspectos do conceito para os quais os alunos apresentam mais dificuldade.

No que diz respeito ao inter-relacionamento entre formas, a combinação de ferramentas simbólicas, incluindo materiais concre- 
tos manipulativos (tais como tijolos de construção) mostraram-se eficazes como precursores dos gráficos no campo conceitual das estruturas aditivas. (SELVA; ROCHA; NUNES, 2005) Esse dado, aliado às discussões feitas por Souza e Seding (SEDIG; SOUZA, 2001), mostra que, no uso das formas, pode haver uma ordem mais adequada na qual elas devam ser expostas ao usuário a fim de favorecer não só experiências diferentes, mas que possuam algum tipo de ordenação e até mesmo de sentido mais adequado entre as experiências. (ELIA; GAGATSIS; DEMETRIOU, 2007)

Nesses estudos anteriores, os autores observam o uso das formas na tentativa de tornar mais claro o papel que cada uma exerce no desenvolvimento das habilidades dos alunos, sozinha ou combinada a outras formas. Isso se justifica no fato de que cada expressão da forma de representação apresenta aspectos diferentes do mesmo conceito e, em conjunto com outras, poderia ampliar a percepção dos alunos para outros aspectos do mesmo conceito ou de conceitos diferentes. Nesse sentido, vê-se que há um esforço em tornar mais claro o alcance ou o desempenho que determinada forma teve sobre tarefas específicas e conceitos específicos.

Nos resultados anteriores, vê-se que a habilidade dos alunos em resolver situações-problema - os erros, as antecipações, ou seja, a organização das ações dos alunos - é influenciada pelas restrições da forma, ou seja, o que eles puderam e o que não puderam fazer no uso dessas. Nesse sentido, as interfaces computacionais interativas vêm ao encontro desses estudos, na tentativa de ampliar as modalidades de interação que o aprendiz pode lançar mão, frente a uma forma de representação, a fim de que, diante de novas possibilidades, nesse caso, os usuários-professores possam perceber de maneira mais ampla as propriedades da forma, características, nuances e especificidades. Em outras palavras, os diferentes tipos de interação ampliam a capacidade mediadora da forma, uma vez que possibilita que outros elementos possam ser percebidos, ou os mesmos, percebidos de outra maneira. (SEDIG; SUMNER, 2006) 
Além dos aspectos relacionados às modalidades de manipulação sobre a forma, faz-se necessário análisar as características, propriedades e dimensões da forma que sejam contíguas aos conceitos por elas representados. (HARRIES; SUGGATE, 2006; SEDIG; KLAWE; WESTROM, 2001; SOUZA; SEDIG, 2001) Por exemplo, Harries e Barmby analisam em Harries e Barmby (2007) as propriedades e características da operação de multiplicação, quais sejam: replicação, uso de operações binárias, comutatividade e distributividade, propondo que a adequação de uma determinada forma para representar o conceitos envolvidos nas operações de multiplicação estaria condicionada à presença de tais caracteristicas. Já Sedig, Klawe e Westrom (2001), em uma interface para o ensino de conceitos de geometria, utilizou um arco de circunferência para representar metaforicamente o conceito de rotação. Na proposta dos autores, o arco utilizado apresenta dois pontos de controle: um é a cabeça do arco, que possibilita a modificação do ângulo de rotação, e outro é centro do arco, que possibilita que o usuário modifique o centro de rotação da imagem. Ou seja, as representações deveriam apresentar um conjunto limitado e compatível de elementos, poderiam capturar as ideias fundamentais do domínio e tais características devem ser passíveis de serem observadas.

Descobrir os elementos da forma que estiveram disponíveis para manipulação, favoreceu que a análise da interação fosse feita em cada uma das partes que compõe a forma de representação utilizada para representar um determinado conceito, e, mais especificamente, relacioná-las às ações dos usuários. Em outras palavras, embora a forma de Vergnaud apresente prevalência de elementos relativos ao conceito de número, o uso de diferentes modalidades de interação aplicada a cada elemento pode favorecer que mesmo aqueles elementos com pouca prevalência sejam manipulados e, por conseguinte, percebidos pelos usuários. 


\section{Influência das regras em ação na emergência de invariantes operatórios}

Cada tarefa executada na interface possui regras que imprimem restrições sobre elas. Por exemplo, a tarefa de "Posicionar" acontece quando o usuário posiciona um elemento no diagrama. No caso da representação aqui utilizada, a tarefa de posicionar pode ser executada no uso do quadrado e do círculo. Entretanto, essa tarefa apresenta restrições associadas à construção do diagrama. Nesse estudo, buscamos identificar a adequação da forma em um contexto de uso, tomando por base tanto a forma de representação quanto o estilo de interação utilizado na manipulação de seus elementos. Nesse sentido, estimamos que proporcionar aos usuários que manipulem outros elementos, notadamente aqueles que ainda não foram manipulados, e por meio de diferentes estilos de interação, irá favorecer tanto a emergência quanto a prevalência de outros invariantes operatórios; contribuindo dessa forma, para ampliar o significado do conceito para o usuário-professor.

\section{Influência da organização das ações dos usuários sobre a interface}

No Quadro 10, são mostradas as contingências observadas na execução da tarefa, ou seja, no momento da execução das tarefas, como as presentes na Quadro 4, os usuários sentiram necessidades ou apresentaram algum tipo de conduta. Essas necessidades, juntamente com o número de ações em cada situação-problema presente no Quadro 8, e com os erros do Quadro 9, são ponto de partida para a compreensão das dificuldades inerentes a cada tarefa. Tal compreensão contribui no refinamento da interface e no ajuste dos tipos de feedback oferecido em cada erro. Nesse sentido, Gagatsis e Elia já haviam apontado (GAGATSIS, 2004) que a habilidade para lidar com a forma interfere na habilidade de resolver uma situação-problema. Num cenário de resolução de problemas, como o aqui apresentado, 
os feedbacks são usados para favorecer que o usuário-professor tenha uma experiência de aprendizagem na qual os erros sejam ponto de partida para reflexão e não apenas fatores impeditivos do seu desempenho.

Ainda no que diz respeito aos erros presentes no Quadro 9, nossos resultados corroboram os já encontrados por Elia, Gagatsis e Demetriou (2007), quanto ao nível de dificuldade das tarefas. Ou seja, os autores confirmaram que os problemas de transformação de medidas com valor desconhecido no início são aqueles considerados mais difíceis. E, por esse motivo, eles indicam a necessidade de se entender os motivos para tal dificuldade. Nesse sentido, a construção dos diagramas oferece meios para fazermos um acompanhamento das ações dos usuários em tarefas e etapas e, nesse estudo, um ambiente computacional é usado para armazenar as tarefas em meios digitais que favoreçam uma análise quantitativa das regularidades observadas, bem como a geração de modelos de usuários. (GOMES; QUEIROZ; CARVALHO, 2003; QUEIROZ; GOMES; CAVALCANTI, 2002) Por exemplo, os erros consecutivos de posicionamento foram cometidos por usuários com mais baixa escolaridade. Eles insistiam em posicionar os elementos na tentativa de tornar a representação do problema semelhante à representação da solução numérica. Já os erros consecutivos de categorização foram cometidos por usuários com mais alta escolaridade. Quando esses usuários tentaram resolver o problema P3, eles insistiram na escolha da categoria de "Composição de medidas".

Nesse estudo, as tarefas P5 e P3 foram aquelas que necessitaram do maior número de ações. Entretanto, vale salientar que dessas 162 ações para P3, como mostrado no Quadro 8, 85 foram executadas por apenas um usuário, ou seja, todos os usuários precisaram de mais ações, mas em apenas um caso houve uma maior discrepância. Os erros no problema P3 foram de posicionamento dos números no diagrama, de sinal e de categorização. Alguns usuários, notadamente aqueles com maior grau de escolaridade, confundiram a transformação aplicada a uma medida com composição de medidas. Ainda sobre 
o problema P3, dos cinco usuários, quatro erraram e necessitaram de mais ações para essa tarefa. Apenas o usuário S1 executou corretamente e não precisou de ajuda. Entretanto, ele já havia resolvido uma situação-problema semelhante. No problema P5, quatro usuários precisaram de ajuda, ocorreram erros de categorização, posicionamento e sinal do número relativo.

Além dos dados presentes no Quadro 8, salienta-se que os problemas P3 e P5 foram apresentados na ordem da numeração apresentada, ou seja, quando os usuários executaram a tarefa P3 e P5, já tinham experiência na execução dos problemas P1, P2 e P4. Em outras palavras, já haviam construído os diagramas para os problemas P1, P2 e P4 e já haviam recebido todos os tipos de feedbacks: instrucional, questionamento, indicativos de sucesso e automatização de passos.

Nos trabalhos relacionados, identificamos que os objetivos estiveram em torno de compreender como se dá o desempenho das crianças quanto às suas habilidades matemáticas, mediante a utilização de diferentes formas de representação, e qual o impacto destas no aprendizado dos alunos, ou seja, como estes apreendem os conceitos matemáticos no uso das formas. Nesse sentido, nosso trabalho se coaduna com Elia, Gagatsis e Demetriou (2007), Harries e Barmby (2007), Harries e Suggate (2006), Michaelidou e Gagatsis (2004) e Selva, Rocha e Nunes (2005) na medida em que o objetivo foi analisar a resolução de situações-problema no uso de uma forma de representação e, mais especificamente, como a flexibilidade de manipulação da forma interfere na sua capacidade mediadora. Entretanto, diferente dos trabalhos correlatos, este estudo foi realizado com participantes adultos, professores do ensino fundamental, que eram integrantes de um curso de especialização no qual foi apresentado a forma de representação discutida pela abordagem de conceito da teoria de Gerard Vergnaud. O material utilizado foi uma interface educativa interativa, o que possibilitou tanto explorar diferentes tipos de manipulação sobre a forma quanto ampliar o contexto da análise, na medida em que foram inseridos elementos da dimensão cognitiva, especifi- 
camente relacionada ao uso de uma determinada tarefa de mouse. Ou seja, além de proporcionar a análise do uso de diferentes tipos de manipulação sobre os elementos da forma, no contexto da interação, ainda foram acrescidos elementos, que revelam a associação entre o uso dessas diferentes tarefas de mouse e as proposições verdadeiras sobre os conceitos expressados pelos usuários.

Em outras palavras, os Quadros 6, 7, 8, 9, 10 e 11 apresentam resultados preliminares, observados a partir da metodologia aqui apresentada, que se diferencia na medida em que buscamos inferir resultados sobre a aprendizagem de conceitos específicos num contexto de uso real, ainda durante a fase incicial de desenvolvimento e buscando envolver o usuário-professor em todo o processo de obtenção dos requisitos funcionais e não funcionais.

Por esse motivo, o Quadro 11 apresenta características que serão utilizados na continuação da interface apresentada na Figura 2. Elas são ponto de partida para a execução de outros testes, a fim de refinar esses resultados ou encontrar outras contingências. O processo de refinamento da interface ocorre de maneira interativa. Nele, todas as modalidades de interação sobre a forma serão analisadas sob a mesma perspectiva. Em razão disso, a interface deve ser modificada rapidamente para agregar as sugestões dos usuários e, em seguida, um outro teste deve acontecer. Nesse ponto, destacamos a importância do protótipo em papel. Além da característica facilitadora da interação, ele ainda pode ser rapidamente modificado, inclusive durante o próprio experimento, permitindo que a sugestões feitas pelos usuários sejam rapidamente agregadas. Nesses momentos, os próprios usuários podem ajudar na construção do protótipo e, por conseguinte, sentirem-se parte integrante do processo.

No que diz respeito à generalidade de tais achados, identificamos a necessidade de proceder a uma análise quantitativa a fim de corroborar os padrões inicialmente identificados qualitativamente. Ainda nesse sentido, pretendemos continuar o estudo a fim de inserir mais participantes com diferentes perfis. No momento, estamos 
trabalhando com 27 participantes, integrantes de um projeto de extensão que recebe apoio do Programa de Bolsas de Integração (Pibin), oferecido pela Universidade Federal do Vale do São Francisco (Univasf). Esse projeto de extensão tem por objetivo principal apresentar aos professores a legenda, os diagramas e o processo de construção dos mesmos.

\section{Conclusões}

A interface mostrada na Figura 2 será concluída no âmbito de um trabalho de conclusão de curso na Univasf, iniciado em agosto de 2010 com conclusão prevista para agosto de 2011. Sua validação iniciou por meio da metodologia aqui adotada (GOMES, 2008), utilizada para verificar aspectos qualitativos sobre aprendizagem de conceitos ainda durante a fase de projeto da interface. Ela será concluída quando a interface em JAVA estiver disponível para testes.

Os professores, participantes do projeto de extensão, estão vinculados a sete escolas de um município baiano. Essas escolas fazem parte de um grupo que apresenta os resultados mais críticos em educação matemática nesse município. Por meio deste estudo, pretendemos não só identificar as necessidades dos professores frente à tarefa de construção dos diagramas, mas, principalmente, colaborar para que eles adquiram experiência na resolução de situações-problema sobre as estruturas aditivas. Além disso, o estado da Bahia é um dos maiores do Brasil e apresenta longas distâncias entre os municípios do interior e os grandes centros. Nesse sentido, a interface aqui refinada será utilizada em programas de educação à distância, favorecendo a professores que estejam afastados dos grandes centros para que possam ter acesso à interfaces computacionais voltadas para aprendizagem de conceitos específicos.

No curso da resolução do problema, o usuário-professor, interagindo com a interface, apresenta um modelo pré-concebido sobre o processo de resolução do problema. Como visto na Figura 4, as formas de representação e o feedback, oferecido pela interface, 
favoreceu tanto o ajuste desse modelo quanto a construção de novos modelos acerca da tarefa em execução. Tais mudanças foram percebidas por meio dos indícios de generalização (QUEIROZ; GOMES, 2008), os quais foram observados nos procedimentos de antecipação e correção de erros sem ajuda.

A exposição desse aprendiz a situações interativas diversas, portanto, irá favorecer o processo de conceitualização, no qual não só um conceito específico será construído, mas as relações que possam haver entre outros conceitos poderão ser percebidas por ele e, por conseguinte, ampliar seus conhecimentos sobre o conteúdo estudado. Para tanto, as situações interativas em uma interface educativa devem ser projetadas com vistas a favorecer a percepção e expansão do significado do conceito para o sujeito, ou, dito de outra forma, é na relação entre as situações interativas que o sujeito e as representações simbólicas que o conceito adquire sentido para esse sujeito. (MOREIRA, 2002) O sentido é, portanto, a rede de esquemas que o sujeito desenvolve para tratar as situações interativas que se apresentam a ele em um contexto específico.

\section{Referências}

ARRIES, T.; BARMBY, P. Representing and understanding multiplication.

Research in Mathematics Education, London, v. 9, n. 1, p. 33-45, abr. 2007.

BAILEY, B. P. et al. Adapting paper prototyping for designing user interfaces for multiple display environments. Personal and Ubiquitous Computing, London, v. 12, n. 3, p. 269-277, fev. 2007.

BRAGA, M. M.; QUEIROZ, A. E. M.; GOMES, A. S. Design de software educacional baseado na teoria dos campos conceituais. In: SIMPÓSIO BRASILEIRO DE INFORMÁTICA EDUCATIVA, 19., 2008, Fortaleza. Anais... Fortaleza: [s.n.], 2008.

ELIA, I.; GAGATSIS, A.; DEMETRIOU, A. The effects of different modes of representation on the solution of one-step additive problems. Learning and Instruction, Oxford, v. 17, n. 6, p. 658-672, dez. 2007.

FLICK, U. Entrevistas semi-estruturadas. In: FLICK, U. Uma introdução à pesquisa qualitativa. 2. ed. Porto Alegre: Bookman, 2004. p. 89-106. 
GAGATSIS, A. A review of the research on the role of external representations on undertanding and learning mathematics and problem solving. Education, [S.I.], n. 2003, p. 2003-2004, 2004.

GAGATSIS, A.; ILIADA, E. A review of some recent studies on the role of representations in mathematics education in cyprus and greece. In: PROCEEDINGS OF THE FOURTH CONGRESS OF THE EUROPEAN SOCIETY FOR RESEARCH IN MATHEMATICS EDUCATION, 4., 2005, Saint Feliux de Guixouls. Anais... Saint Feliux de Guixouls: CERME, 2005.p. 102-111. Disponível em: <http://ermeweb.free.fr/CERME4/CERME4_WG1.pdf\#page=38>. Acesso em: 5 jun. 2012.

GOLDIN, G. Representations and the psychology of mathematics education. The Journal of Mathematical Behavior, Montreal, v. 17, n. 1, p. 1-4, 1998a.

GOLDIN, G. Representations and the psychology of mathematics education: part II. The Journal of Mathematical Behavior, . Montreal, v. 17, n. 2, p. 135, 1998b.

GOLDIN, G. A. Representation in school mathematics: a unifying perspective. In: KILPATRICK, J.; MARTIN, W. G.; SCHIFTER, D. (Ed.). Research companion to principles and standards for school mathematics Reston: National Council of Teachers of Mathematics, 2003. p. 275-285.

GOLDIN, G.; KAPUT, J. A joint perspective on the idea of representation in learning and doing mathematics. In: STEFFE, L. P.; NESHER, P. (Ed.). Theories of mathematical learning. Mahwah: Lawrence Erlbaum Associates, 1996. p. 397-430.

GOMES, A. S. Referencial teórico construtivista para avaliação de software educativo. Revista Brasileira de Informática na Educação, Florianópolis, v. 16, n. 2, p. 9-21, 2008.

GOMES, A. S.; QUEIROZ, A. E. de M.; CARVALHO, F. de A. T. User learning Modeling in learnware design - Case study with dynamic geometry software. In: JACKO, J. ; STEPHANIDIS, C. (Ed.). Human computer interaction. Mahwah: Lawrence Erlbaum, 2003. (Proceeding's of HCl International 2003, v. 2).

HARRIES, T.; SUGGATE, J. Exploring links across representations of numbers with young children. International Journal for Technology in Mathematics Education, Hertfordshire,v. 13, n. 2, p. 53-64, jun. 2006.

KAPUT, J. The representational roles of technology in connecting mathematics with authentic experience. In: BIEHLER, R.; W. SCHOLZ, R.; STRÄSSE, R.; 
WINKELMANN, B. (Ed.). Didactics of mathematics as a scientific discipline. Dordrecht: Kluwer, 1993. p. 379-393.

LIANG, H. N.; SEDIG, K. Role of interaction in enhancing the epistemic utility of $3 \mathrm{~d}$ mathematical visualizations. International journal of computers for mathematical Learning, Dordrecht, v. 15, n. 3, p. 191-224, Aug. 2010.

MICHAELIDOU, N.; GAGATSIS, A. The number line as a representation of decimal numbers: A research with sixth grade students. In: CONFERENCE OF THE INTERNATIONAL GROUP FOR THE PSYCHOLOGY OF MATHEMATICS EDUCATION, 28., 2004, Bergen. Anais... Bergen: PME, 2004. p. 305-312. Disponível em: <http:// www.emis.ams.org/proceedings PME28/RR/ RR208_ Michaelidou.pdf>. Acesso em: 5 jun. 2012.

MOREIRA, M. A. A Teoria dos campos conceituais de vergnaud, o ensino de ciências e pesquisa nesta área. Investigações em Ensino de Ciências, Porto Alegre, v. 7, n. 1, p. 7-29, 2002.

QUEIROZ, A. E. de M.; GOMES, A. S. Efeitos da manipulação direta reflexiva na resolução de problemas sobre estruturas aditivas. Revista Brasileira de Informática Educativa, Rio de Janeiro, v. 16, n. 2, 2008.

QUEIROZ, A. E. de M.; GOMES, A. S.; CAVALCANTI, F. de A. T. Mineração de Dados de IHC para Interfaces Educativas. [S.I.], 2002.

SEDIG, K.; KLAWE, M.; WESTROM, M. Role of interface manipulation style and scaffolding on cognition and concept learning in learnware. ACM Transactions on Computer-Human Interaction, New York, v. 8, n. 1, p. 34-59, mar. 2001.

SEDIG, K.; SUMNER, M. Characterizing interaction with visual mathematical representations. International Journal of Computers for Mathematical Learning, Dordrecht, v. 11, n. 1, p. 1-55, jul. 2006.

SELVA, A. C. V.; ROCHA, J. T. DA; NUNES, T. Solving additive problems at pre-elementary school level with the support of graphical representation. In: CONFERENCE OF THE INTERNATIONAL GROUP FOR THE PSYCHOLOGY OF MATHEMATICS EDUCATION, 28., 2005., Melbourne. Anais... Melbourne: [s.n.], 2005. Disponível em: <http://www.emis.ams.org/proceedings/PME29/ PME29CompleteProc/PME29Vol4Mul_Wu.pdf\#page=167>. Acesso em: 5 jun. 2012.

SHNEIDERMAN, B. Designing the user interface. Reading: Addison Wesley Longman, 1998. 
SNYDER, C. Paper prototyping: the fast and easy way to design and refine user interfaces. San Francisco: Morgan Kaufmann Publishers, 2003.

SOUZA, C. S. de; SEDIG, K. Semiotic considerations on direct concept manipulation as a distinct interface style for learnware. In: WORKSHOP DE FATORES HUMANOS EM SISTEMAS COMPUTACIONAIS, 4., 2001, Porto Alegre. Anais... Porto Alegre: SBC, 2001. p. 229-241. Disponível em: <http://citeseerx. ist.psu.edu/viewdoc/download?doi=10.1.1.88.412\&rep=rep1\&type=pdf $>$. Acesso em: 5 jun. 2012.

VERGNAUD, G. Psicologia do desenvolvimento cognitivo e didática das matemáticas Um exemplo. Análise Psicológica, Lisboa, p. 75-90, 1986.

VERGNAUD, G. A comprehensive theory of representation for mathematics education. The Journal of Mathematical Behavior, Montreal, v. 17, n. 2, p. 167-181, 1998.

WOOD, D.; BRUNNER, J.; ROSS, G. The role of tutoring in problem solving. Jornaul of Child Psychology and Psychiatry and Allied Disciplines, Cambridge, n. 17, p. 89-100, 1971. 